\title{
Teneurin Structure: Splice Variants of a Bacterial Toxin Homolog Specifies Synaptic Connections
}

\author{
Demet Araç ${ }^{1,2 *}$ and Jingxian $\mathrm{Li}^{1,2}$ \\ ${ }^{1}$ Department of Biochemistry \& Molecular Biology, The University of Chicago, Chicago, IL, United States, ${ }^{2}$ Grossman \\ Institute for Neuroscience, Quantitative Biology and Human Behavior, The University of Chicago, Chicago, IL, United States
}

OPEN ACCESS

Edited by:

Antony Boucard Jr.

Center for Research and Advanced Studies of the National Polytechnic Institute (CINVESTAV), Mexico

Reviewed by:

Davide Comoletti,

Rutgers University, The State University of New Jersey,

United States

Susanne Ressl,

Indiana University Bloomington,

United States

*Correspondence:

Demet Araç

arac@uchicago.edu

Specialty section:

This article was submitted to Neuroendocrine Science,

a section of the journal

Frontiers in Neuroscience

Received: 24 April 2019

Accepted: 26 July 2019

Published: 07 August 2019

Citation:

Araç D and Li J (2019) Teneurin

Structure: Splice Variants of a Bacterial Toxin Homolog Specifies

Synaptic Connections.

Front. Neurosci. 13:838.

doi: 10.3389/fnins.2019.00838
Teneurins are a conserved family of cell-surface adhesion molecules that mediate cellular communication, and play key roles in embryonic and neural development. Their mechanisms of action remained unclear due in part to their unknown structures. In recent years, the structures of teneurins have been reported at atomic resolutions and revealed a clear homology to bacterial Tc toxins with no similarity to other eukaryotic proteins. Another surprising observation was that alternatively spliced variants of teneurins interact with distinct ligands, and thus specify excitatory vs. inhibitory synapses. In this review, we discuss teneurin structures that together with structureguided biochemical and functional analyses, provide insights for the mechanisms of trans-cellular communication at the synapse and other cell-cell contact sites.

Keywords: teneurin, ODZ, adhesion GPCR, latrophilin/ADGRL, synapse, structure, alternative splicing, FLRT

The coupling of extracellular adhesion to intracellular signaling is essential for cells to interpret cues from neighboring cells. With four members in humans, teneurins (TENs) are evolutionarily conserved cell-adhesion proteins that mediate intercellular communication (Baumgartner et al., 1994; Levine et al., 1994; Lossie et al., 2005; Tucker and Chiquet-Ehrismann, 2006; Nakamura et al., 2013; Mosca, 2015). Recent studies show that TENs play central roles in tissue polarity, embryogenesis, heart development, axon guidance, and synapse formation (Levine et al., 1994; Doyle et al., 2006; Silva et al., 2011; Hong et al., 2012; Mosca et al., 2012; Boucard et al., 2014; Mosca and Luo, 2014; Woelfle et al., 2016; Berns et al., 2018). Genetic studies link them to various diseases including neurological disorders, developmental problems, various cancers and congenital general anosmia (Aldahmesh et al., 2012; Ziegler et al., 2012; Hor et al., 2015; Alkelai et al., 2016; Chassaing et al., 2016; Graumann et al., 2017; Talamillo et al., 2017). TENs are type-II transmembrane proteins with large (>2000 amino acids) C-terminal extracellular regions (ECRs) that mediate heterophilic and homophilic trans-cellular interactions that are key for various functions. Most TEN ECRs do not exhibit readily identifiable domains by sequence analysis. Despite the central importance of TENs in multiple physiological functions, the lack of information on the structure of TENs and a molecular understanding of TEN interactions with heterophilic or homophilic ligands has been one of the limiting factors in delineating the mechanisms of TEN action.

\section{TENEURIN IS HOMOLOGOUS TO BACTERIAL TOXINS}

TENs are composed of an $\mathrm{N}$-terminal cytoplasmic tail, a single transmembrane region (TM), and a large ECR (Figure 1A; Baumgartner and Chiquet-Ehrismann, 1993; Baumgartner et al., 1994; Levine et al., 1994; Oohashi et al., 1999). The ECR of TENs comprises eight epidermal growth factor (EGF) motifs that are followed by the large unknown region composed of domains identified 
as 2, 3, 4 and 5 in Figure 1A. TENs form cis-dimers via conserved disulfide bonds formed between their EGF repeats juxtaposed to the transmembrane helix (Oohashi et al., 1999; Feng et al., 2002; Vysokov et al., 2016; Figure 2). Recently, important breakthroughs were achieved and the high resolution cryo-electron microscopy structures of the unknown region from human TEN2, mouse TEN3 and the crystal structure of chicken TEN2 have been reported revealing a highly unusual structure (Jackson et al., 2018; Li et al., 2018). The structures agree with each other and show that the TEN YD repeats corresponding to domain 4 has a striking structural similarity to that of bacterial Tc-toxins as previously suspected (Tucker et al., 2012; Busby et al., 2013; Meusch et al., 2014; Figure 1B). Surprisingly, the TEN2 ECR has an unusual architecture that has not been observed in any other eukaryotic protein before (Figures 1B-D). The structure comprises a large cylindrical $\beta$-barrel (blue) sealed at the bottom by an Immunoglobulin (Ig)-like domain (yellow) and a $\beta$-propeller (green); and at the top by a C-terminal domain (magenta).

The TEN $\beta$-barrel has high homology to bacterial Tc-toxins (Figures 1B,E). Bacterial Tc-toxins are secreted proteins and comprise a large barrel that contains a toxic domain (Landsberg et al., 2011; Busby et al., 2013; Meusch et al., 2014; Figure 1E). The toxin domain is autocleaved from the rest of the protein,

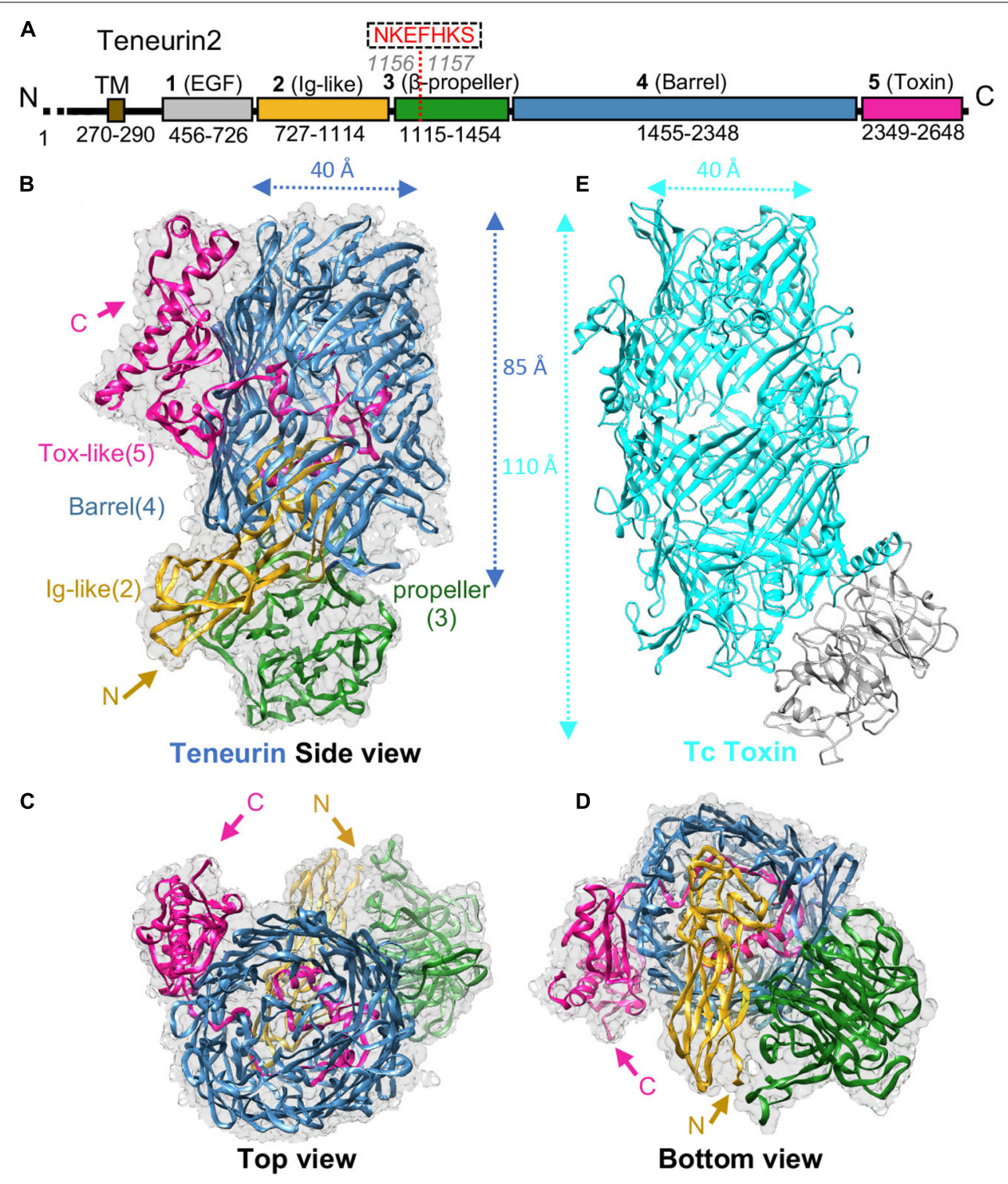

FIGURE 1 | TEN structure reveals homology to bacterial Tc toxins. (A) Scheme for human TEN2. Domains are colored gray, yellow, green, blue, and magenta for domains 1-5, respectively; transmembrane region (TM) in colored brown. Residue numbers for each domain are indicated. (B-D) Cryo-EM structure of TEN2 ECR (2-5) is shown from different views (Li et al., 2018) (PDB ID: 6CMX). (E) The TEN2 domain 4 (blue) is a barrel that is homologous to the BC components of bacterial Tc-toxins (TcC-toxin, cyan, PDB ID: 4O9X). Figure modified from Li et al. (2018). 
passes through the barrel tunnel and is injected into the infected cell to execute its toxic effects usually by binding to the infected cell's DNA or other components (Figure 1E). The $\beta$-barrel of TEN2 structure partially encapsulates a C-terminal toxin-like domain. The toxin-like domain, however, exits the barrel from an opening and is tethered to the outer surface of the barrel (Li et al., 2018; Figure 1B). In spite of structural differences, the autoproteolytic site of the bacterial Tc-toxin is conserved in the TEN2 structure (Li et al., 2018; Arac and Li, 2019) (see Li et al., 2018 and Araç and Li, Current Opinion in Structural Biology, 2019 for in-depth structural analysis and comparison of TEN and bacterial Tc toxin structures). These observations raised numerous questions and exciting possibilities about how TENs may function. An immediate question is whether TENs function similar to bacterial toxins. Is the toxin-like domain of TEN autocleaved, released and inserted into the neighbor cells? Is it toxic to neighbor cells? Does it go into the nucleus and bind to DNA or other components of the cells? Does it mediate intracellular signaling or induce synapse formation and developmental changes? Does the previously reported C-terminal peptide of TEN act like a neuropeptide (Wang et al., 2005; Vysokov et al., 2016)? These are very exciting and open-ended questions that need to be answered in the future.

\section{ALTERNATIVE SPLICING REGULATES TENEURIN INTERACTIONS}

Recent studies provided further surprising results about the roles of splice variants of TENs. TENs are alternatively spliced at two sites within the ECR and include nine- and sevenresidue insertions at the EGF repeats and the $\beta$-propeller regions, respectively (Tucker et al., 2001). The extracellular region of TENs mediates homophilic and heterophilic interactions that have specific roles in different functions of TENs, however the role of alternative splicing in mediating these interactions remained unknown (Leamey and Sawatari, 2014; Woelfle et al., 2016; Sudhof, 2017). The high-affinity heterophilic interaction of TENs with latrophilins (LPHN1-3), a family of adhesion G Protein Coupled Receptors (GPCRs) that have key roles in synapse development and embryogenesis, regulates synapse formation and organization (Silva et al., 2011; Boucard et al., 2014). On the other hand, trans-homodimerization of TENs is reported to be critical for correct matching of axons with their partner dendrites, a process that is critical for neural circuit-wiring in the nervous system (Hong et al., 2012; Mosca et al., 2012; Mosca, 2015).

Surprisingly, Li et al. (2018) showed that an alternatively spliced seven-residue region (NKEFKHS) within the $\beta$-propeller

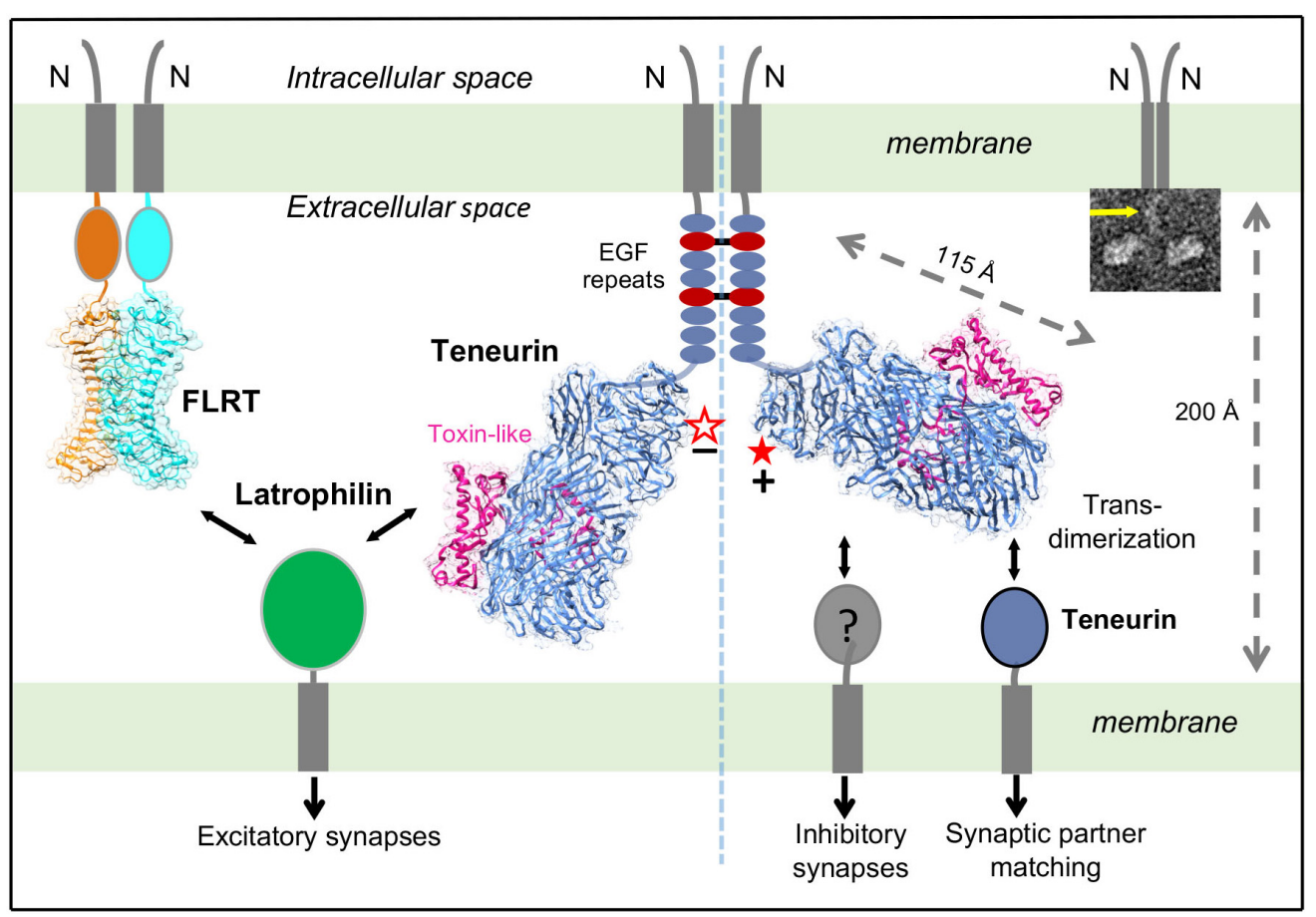

FIGURE 2 | Different splice variants of TENs regulate distinct synapse specifications. Alternative splicing is a molecular switch to regulate which binding partner TEN2 binds to, and what respective function TEN2 does. On the left side, the -SS isoform of TEN2 (empty star) interacts with LPHN. When it is co-expressed with FLRT, TEN2 isoform that lacks the splice insert induces excitatory postsynaptic differentiation. On the right side, TEN2 that includes the splice insert (full star) cannot interact with LPHN, but it can form trans-homodimers to mediate neural circuit-wiring and induces inhibitory synapses. The left and right sides of the TEN2 dimer represent excitatory and inhibitory synapses, respectively. The membranes, teneurin structure (Li et al., 2018) (PDB ID: 6CMX), and distance between the membranes are drawn to scale. Alternative splice site is shown by red star. Insert: Representative negative stain EM micrographs of TEN2 ECR(1-5) shows a TEN2 -SS cis-dimer. Yellow arrows indicate EGF repeats. Figure modified from Li et al. (2018). 
acts as a switch to regulate trans-cellular adhesion of TEN2 to LPHNs (Figure 2, red star). The splice variant that lacks the seven amino acids can bind to full-length LPHN presented on the neighbor cells in cell-aggregation assays and this interaction activates trans-cellular signaling in a LPHN-dependent manner (Figure 2, left side). The other splice variant that includes the seven amino acids, however, is unable to interact with full-length LPHN in identical experiments (Figure 2, right side). Similarly, the same alternatively spliced site that regulates the TEN/LPHN interaction has been reported to also regulate TEN transhomodimerization (Berns et al., 2018). Intriguingly, the splice variant that lacks these seven amino acids cannot mediate transhomodimerization, whereas the other variant can (Figure 2, right side). Taken together, these results suggest that TEN splice variants can either interact with LPHNs or mediate transdimerization with itself, but a single variant cannot mediate both interactions.

\section{DIFFERENT SPLICE VARIANTS OF TENS PERFORM DISTINCT SYNAPSE SPECIFICATIONS}

As different TEN splice variants are involved in different ligand interactions, the next question arose: Do different TEN splice variants perform biologically distinct functions? Subsequent studies by $\mathrm{Li}$ et al. (2018) examined the capability of TEN2 + SS and TEN2 -SS to induce artificial synapse formation. In these assays, HEK293 cells expressing TEN variants were co-cultured with primary neurons; and inhibitory and excitatory synapse formation was monitored for pre- and post-synaptic differentiation for both types of synapses ( $\mathrm{Li}$ et al., 2018). The results showed that TEN2 + SS induced GABAergic (inhibitory) synaptic specializations but failed to induce glutamatergic (excitatory) synaptic specifications (Figure 2, right side; Li et al., 2018).

\section{REFERENCES}

Aldahmesh, A. M., Mohammed, J. Y., Al-Hazzaa, S., and Alkuraya, S. F. (2012). Homozygous null mutation in ODZ3 causes microphthalmia in humans. Genet. Med. 14, 900-904. doi: 10.1038/gim.2012.71

Alkelai, A., Olender, T. R., Haffner-Krausz, M., Soory, T. M., Boyko, V., Tatarskyy, P., et al. (2016). A role for TENM1 mutations in congenital general anosmia. Clin. Genet. 90, 211-219. doi: 10.1111/cge.12782

Arac, D., and Li, J. (2019). Teneurins and latrophilins: two giants meet at the synapse. Curr. Opin. Struct. Biol. 54, 141-151. doi: 10.1016/j.sbi.2019.01.028

Baumgartner, S., and Chiquet-Ehrismann, R. (1993). Tena, a Drosophila gene related to tenascin, shows selective transcript localization. Mech. Dev. 40, 165-176. doi: 10.1016/0925-4773(93)90074-8

Baumgartner, S., Martin, D., Hagios, C., and Chiquet-Ehrismann, R. (1994). Tenm, a Drosophila gene related to tenascin, is a new pair-rule gene. EMBO J. 13, 3728-3740. doi: 10.1002/j.1460-2075.1994.tb06682.x

Berns, D. S., DeNardo, L. A., Pederick, D. T., and Luo, L. (2018). Teneurin3 controls topographic circuit assembly in the hippocampus. Nature 554, 328-333. doi: 10.1038/nature25463
On the other hand, initially, TEN2 -SS failed to recruit both excitatory and inhibitory synaptic markers (Li et al., 2018). However, when fibronectin leucine rich repeat transmembrane protein (FLRT3), another LPHN ligand that on its own is unable to induce pre- or postsynaptic specializations, was coexpressed in HEK293T cells with the TEN2 -SS, these molecules together potently induced excitatory but not inhibitory postsynaptic specializations (Sando et al., 2019; Figure 2, left side). The other splice variant, TEN + SS (that cannot bind LPHN and cannot induce excitatory synapses) was still inactive in excitatory synapse formation, even when coexpressed with FLRT (Sando et al., 2019). Thus, TEN -SS and FLRT can stimulate excitatory synapse formation in combination, but not separately. These results are consistent with studies in transgenic LPHN mice in vivo, where coincident binding of both TEN and FLRT to LPHN is required for excitatory synapse formation (Sando et al., 2019).

As we start to understand the functions of TENs, these observations are likely only a glimpse of the complexity of the TEN system. Many questions arise: How can a seven-residue splice site affect ligand binding that is away from the ligand binding site on TEN (Figure 2, left side; Li et al., 2018). What are the implications of various TEN variants and isoforms for neural wiring? Do other splice variants have important biological functions? What are the other unknown ligands for TENs? What is the role of TEN/LPHN interaction in other systems such as embryonic development? TEN field is awaiting further exciting and surprising findings.

\section{AUTHOR CONTRIBUTIONS}

Both authors wrote the manuscript.

\section{FUNDING}

This work was supported by grants R01 GM120322 and R01 GM134035-01 to DA and the American Heart Association grant \#19POST34380439/Jingxian Li/2019-2020.

Boucard, A. A., Maxeiner, S., and Sudhof, C. T. (2014). Latrophilins function as heterophilic cell-adhesion molecules by binding to teneurins: regulation by alternative splicing. J. Biol. Chem. 289, 387-402. doi: 10.1074/jbc.M113.504779

Busby, N. J., Panjikar, S., Landsberg, J. M., Hurst, R. M., and Lott, S. J. (2013). The BC component of ABC toxins is an RHS-repeat-containing protein encapsulation device. Nature 501, 547-550. doi: 10.1038/nature12465

Chassaing, N., Ragge, N., Plaisancie, J., Patat, O., Genevieve, D., Rivier, F., et al. (2016). Confirmation of TENM3 involvement in autosomal recessive colobomatous microphthalmia. Am. J. Med. Genet. A 170, 1895-1898. doi: 10.1002/ajmg.a.37667

Doyle, S. E., Scholz, M. J., Greer, K. A., Hubbard, A. D., Darnell, D. K., Antin, P. B., et al. (2006). Latrophilin-2 is a novel component of the epithelial-mesenchymal transition within the atrioventricular canal of the embryonic chicken heart. Dev. Dyn. 235, 3213-3221. doi: 10.1002/dvdy.20973

Feng, K. X., Zhou, H., Oohashi, T., Morgelin, M., Lustig, A., Hirakawa, S., et al. (2002). All four members of the Ten-m/Odz family of transmembrane proteins form dimers. J. Biol. Chem. 277, 26128-26135. doi: 10.1074/jbc.m203722200

Graumann, R., Di Capua, G. A., Oyarzun, E. J., Vasquez, M. A., Liao, C., Branes, A. J., et al. (2017). Expression of teneurins is associated with tumor 
differentiation and patient survival in ovarian cancer. PLoS One 12:e0177244. doi: 10.1371 /journal.pone.0177244

Hong, W., Mosca, T. J., and Luo, L. (2012). Teneurins instruct synaptic partner matching in an olfactory map. Nature 484, 201-207. doi: 10.1038/nature10926

Hor, H., Francescatto, L., Bartesaghi, L., Ortega-Cubero, S., Kousi, M., LorenzoBetancor, O., et al. (2015). Missense mutations in TENM4, a regulator of axon guidance and central myelination, cause essential tremor. Hum. Mol. Genet. 24, 5677-5686. doi: 10.1093/hmg/ddv281

Jackson, A. V., Meijer, H. D., Carrasquero, M., van Bezouwen, L., SLowe, D. E., Kleanthous, C., et al. (2018). Structures of Teneurin adhesion receptors reveal an ancient fold for cell-cell interaction. Nat. Commun. 9:1079. doi: 10.1038/ s41467-018-03460-0

Landsberg, J. M., Jones, A. S., Rothnagel, R., Busby, N. J., Marshall, D. S., Simpson, M. R., et al. (2011). 3D structure of the Yersinia entomophaga toxin complex and implications for insecticidal activity. Proc. Natl. Acad. Sci. U.S.A. 108, 20544-20549. doi: 10.1073/pnas.1111155108

Leamey, A. C., and Sawatari, A. (2014). The teneurins: new players in the generation of visual topography. Semin. Cell Dev. Biol. 35, 173-179. doi: 10. 1016/j.semcdb.2014.08.007

Levine, A., Bashan-Ahrend, A., Budai-Hadrian, O., Gartenberg, D., Menasherow, S., and Wides, R. (1994). Odd Oz: a novel Drosophila pair rule gene. Cell 77, 587-598. doi: 10.1016/0092-8674(94)90220-8

Li, J., Shalev-Benami, M., Sando, R., Jiang, X., Kibrom, A., Wang, J., et al. (2018). Structural basis for teneurin function in circuit-wiring: a toxin motif at the synapse. Cell 173, 735-748.e15. doi: 10.1016/j.cell.2018.03.036

Lossie, A. C., Nakamura, H., Thomas, S. E., and Justice, M. J. (2005). Mutation of $17 \mathrm{Rn} 3$ shows that Odz4 is required for mouse gastrulation. Genetics 169, 285-299. doi: 10.1534/genetics.104.034967

Meusch, D., Gatsogiannis, C. R., Efremov, G., Lang, E. A., Hofnagel, O., Vetter, R. I., et al. (2014). Mechanism of Tc toxin action revealed in molecular detail. Nature 508, 61-65. doi: 10.1038/nature13015

Mosca, T. J. (2015). On the Teneurin track: a new synaptic organization molecule emerges. Front. Cell. Neurosci. 9:204. doi: 10.3389/fncel.2015.00204

Mosca, T. J., Hong, W., Dani, V. S., Favaloro, V., and Luo, L. (2012). Trans-synaptic Teneurin signalling in neuromuscular synapse organization and target choice. Nature 484, 237-241. doi: 10.1038/nature10923

Mosca, T. J., and Luo, L. (2014). Synaptic organization of the Drosophila antennal lobe and its regulation by the Teneurins. eLife 3:e03726. doi: 10.7554/eLife. 03726

Nakamura, H., Cook, R. N., and Justice, M. J. (2013). Mouse Tenm4 is required for mesoderm induction. BMC Dev. Biol. 13:9. doi: 10.1186/1471-213X-13-9

Oohashi, T. X., Zhou, H., Feng, K., Richter, B., Morgelin, M., Perez, M. T., et al. (1999). Mouse ten-m/Odz is a new family of dimeric type II transmembrane proteins expressed in many tissues. J. Cell Biol. 145, 563-577. doi: 10.1083/jcb. 145.3.563

Sando, R., Jiang, X., and Sudhof, C. T. (2019). Latrophilin GPCRs direct synapse specificity by coincident binding of FLRTs and teneurins. Science 363:eaav7969. doi: $10.1126 /$ science.aav7969
Silva, J. P., Lelianova, G. V., Ermolyuk, S. Y., Vysokov, N., Hitchen, P. G., Berninghausen, O., et al. (2011). Latrophilin 1 and its endogenous ligand Lasso/teneurin-2 form a high-affinity transsynaptic receptor pair with signaling capabilities. Proc. Natl. Acad. Sci. U.S.A. 108, 12113-12118. doi: 10.1073/pnas. 1019434108

Sudhof, C. T. (2017). Synaptic neurexin complexes: a molecular code for the logic of neural circuits. Cell 171, 745-769. doi: 10.1016/j.cell.2017. 10.024

Talamillo, A., Grande, L., Ruiz-Ontanon, P., Velasquez, C., Mollinedo, P., Torices, S., et al. (2017). ODZ1 allows glioblastoma to sustain invasiveness through a Myc-dependent transcriptional upregulation of RhoA. Oncogene 36, 1733 1744. doi: 10.1038 /onc. 2016.341

Tucker, P. R., Beckmann, J., Leachman, T. N., Scholer, J., and Chiquet-Ehrismann, R. (2012). Phylogenetic analysis of the teneurins: conserved features and premetazoan ancestry. Mol. Biol. Evol. 29, 1019-1029. doi: 10.1093/molbev/ msr271

Tucker, P. R., Chiquet-Ehrismann, R., Chevron, P. M., Martin, D., Hall, J. R., and Rubin, P. B. (2001). Teneurin-2 is expressed in tissues that regulate limb and somite pattern formation and is induced in vitro and in situ by FGF8. Dev. Dyn. 220, 27-39. doi: 10.1002/1097-0177(2000)9999:9999<::aid-dvdy1084>3. $0 . \mathrm{co} ; 2-\mathrm{b}$

Tucker, R. P., and Chiquet-Ehrismann, R. (2006). Teneurins: a conserved family of transmembrane proteins involved in intercellular signaling during development. Dev. Biol. 290, 237-245. doi: 10.1016/j.ydbio.2005.11.038

Vysokov, V. N., Silva, P. J. V., Lelianova, G., Ho, C. M., Djamgoz, B., Tonevitsky, G. A., et al. (2016). The mechanism of regulated release of Lasso/Teneurin-2. Front. Mol. Neurosci. 9:59. doi: 10.3389/fnmol.2016.00059

Wang, L., Rotzinger, S., Al Chawaf, A., Elias, F. C., Barsyte-Lovejoy, D., Qian, X., et al. (2005). Teneurin proteins possess a carboxy terminal sequence with neuromodulatory activity. Brain Res. Mol. Brain Res. 133, 253-265. doi: 10. 1016/j.molbrainres.2004.10.019

Woelfle, R., D'Aquila, A. L., and Lovejoy, A. D. (2016). Teneurins, TCAP, and latrophilins: roles in the etiology of mood disorders. Transl. Neurosci. 7, 17-23. doi: 10.1515/tnsci-2016-0004

Ziegler, A., Corvalan, A., Roa, I. J., Branes, A., and Wollscheid, B. (2012). Teneurin protein family: an emerging role in human tumorigenesis and drug resistance. Cancer Lett. 326, 1-7. doi: 10.1016/j.canlet.2012.07.021

Conflict of Interest Statement: The authors declare that the research was conducted in the absence of any commercial or financial relationships that could be construed as a potential conflict of interest.

Copyright (C) 2019 Araç and Li. This is an open-access article distributed under the terms of the Creative Commons Attribution License (CC BY). The use, distribution or reproduction in other forums is permitted, provided the original author(s) and the copyright owner(s) are credited and that the original publication in this journal is cited, in accordance with accepted academic practice. No use, distribution or reproduction is permitted which does not comply with these terms. 